Moore, W. T. \& Mason, B. (1951). J. gen. Microbiol. 5, 516-518.

\title{
Blender for Preparation of Mycelial Inocula
}

\author{
By W. T. MOORE AND B. MASON \\ Butterwick Research Laboratories, Imperial Chemical Industries Ltd., \\ Welroyn, Herts
}

SUMMARY: In this blender the container and cutter assembly can be removed for heat sterilization. A cotton-wool gland maintains sterility during blending.

For certain types of mycological work undertaken in these laboratories it has been found convenient to use mycelial inocula prepared by breaking up mycelial mats, from surface cultures, in a blender. For such purposes a blender is needed which can be heat-sterilized before use and in which sterility can be maintained during use. For this purpose we have developed, and used for 3 years, a blender which we consider may have wider applications.

The commercial blenders of our experience are unsuitable. Bottom drive blenders cannot be kept clean without stripping the gland bearing. Nor can blenders with a self-lubricated bearing be repeatedly heat-sterilized because this sweats out the oil from the sintered metal. Top drive blenders do not allow for the sterilization of the shaft in the container. Moreover, in most blenders there is no provision for blending small quantities.

The container of our blender (Fig. 1) is shaped to deal effectively with quantities from 20 to $200 \mathrm{ml}$. Baffle strips are attached to the wall to decrease the spinning of the liquid. The lid screws on, and the container is held in alinement with the shaft and motor by screwing into the base of the stand. Container, lid, shaft and cutters are made of stainless steel. To allow the upper end of the shaft to be inserted into the coupling, the electric motor which runs at 6000 r.p.m. can be raised by a screw thread. The square supporting column ensures alinement of the cutters in the centre of the container. The gland is packed with cotton-wool before sterilization. The inside of the gland has a right-hand thread and this, with the clockwise rotation of the shaft, keeps the cotton-wool in position during blending. The direction of rotation also tends to tighten the right-hand thread of the shaft coupling.

The sterilized container with shaft is screwed into the base with the motor raised. The halves of the coupling are screwed together and the motor lowered.

Two minutes' blending time is usually sufficient.

There are no mechanical hazards, because the container surrounds the cutters when they are moving.

\section{Tests of sterility during operation}

The sterilized blender containing $50 \mathrm{ml}$. of sterile nutrient broth was run for $30 \mathrm{~min}$. During the last $5 \mathrm{~min}$. the top of the container was sprayed with a 24. hr. broth culture of Serratia marcescens to ensure a heavily contaminated atmosphere. No colonies appeared on any test plate after 5 days' incubation.

On no occasion have we had evidence that living mycelium is driven out of the container during operation of the blender. 


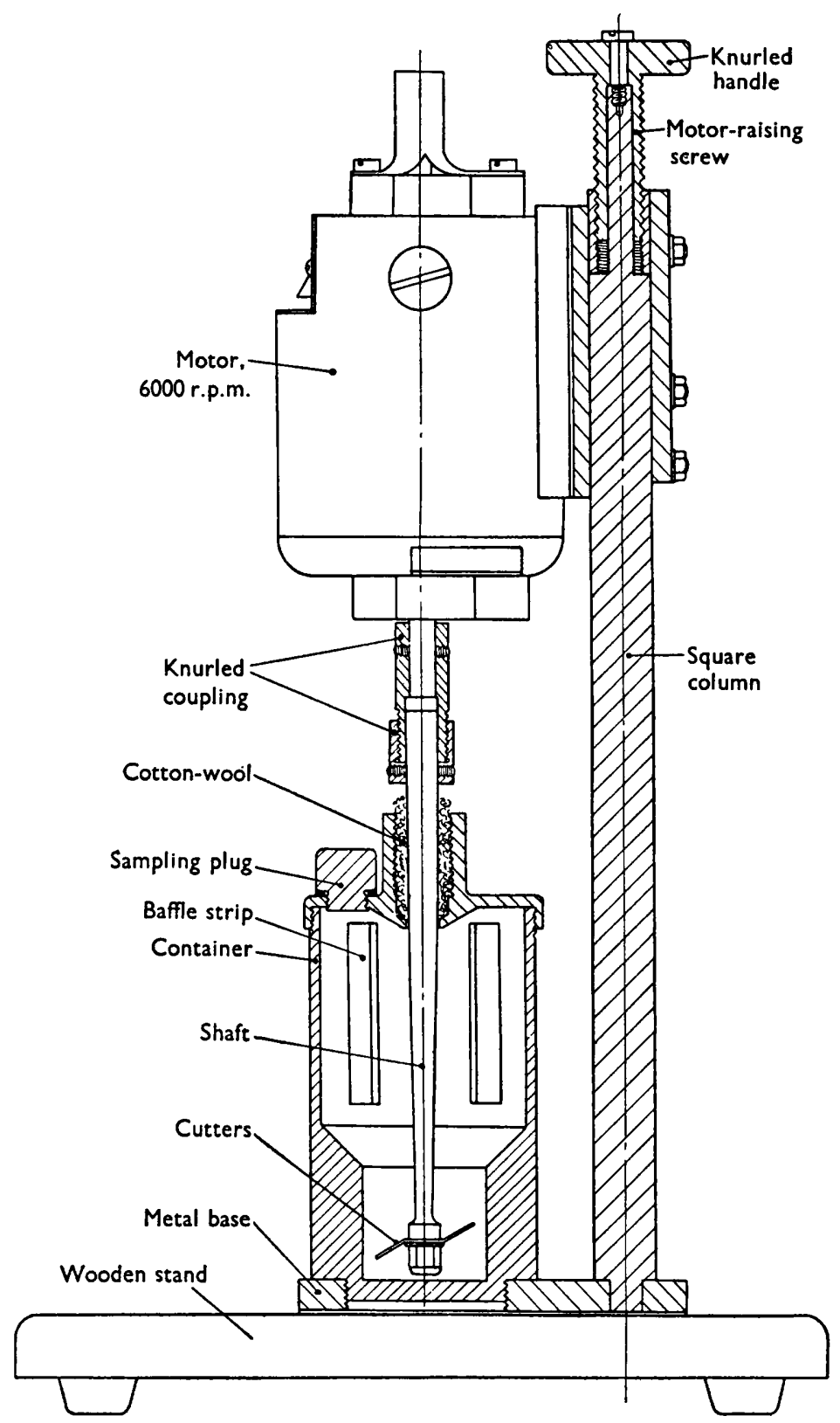

Fig. 1

Details of construction

Container. This is machined to $2 \frac{3}{4}$ in. O.D. from a solid bar of Firth Vickers E.M.S. Staybrite. The four $\frac{3}{8} \times \frac{1}{8}$ in. baffle strips are each pressed on with two $\frac{1}{18}$ in. diameter dowel pins. The internal depth is $4 \frac{1}{2} \mathrm{in}$. and the internal diameters $2 \frac{1}{2}$ and $1 \frac{1}{2}$ in. 
Cover. Machined from the same material as the container. The total depth is $1 \frac{3}{8}$ in. and the outer edge is knurled. The 1 in. diameter projection is screwed internally $\frac{3}{4}$ in. B.S.F.; the diametrical clearance of the hole on the shaft being $\frac{1}{32}$ in. $\mathbf{A} \frac{1}{2}$ in. B.S.F. plug and rubber washer seal the sampling hole. A rubber washer may be inserted between the container and the cover but we have not found this necessary.

Shaft assembly. The tapered shaft is $\frac{3}{8}$ in. diameter at the coupling. Two cutters registering on two flats on the shaft are secured by a nut. One cutter is straight and the other bent, and the leading edges of both are sharp. The half coupling is in true alinement with the shaft and secured to it by two grub screws. All these parts are made of Staybrite.

Motor. This is a series-wound $\frac{1}{80}$ h.p., 240 V. a.c., s.P. motor, rated at 2000 r.p.m. This speed is for the motor fully loaded, and under the light loading in the blender it runs at approximately 6000 r.p.m.

Stand. A brass plate supporting a $\frac{13}{18}$ in. square column is screwed to a wooden base mounted on rubber feet. The motor bracket, incorporating a nut, is a good sliding fit on the column. The knurled handle with screw is attached to the top of the column but is free to rotate.

This blender can easily be made in a laboratory machine shop and the authors will be pleased to supply full constructional details to those interested.

We thank Miss Anne Levy for her most useful criticisms and for the sterility tests described.

(Received 18 December 1950) 
Journal of General Microbiology, Vol. 5, No. 3

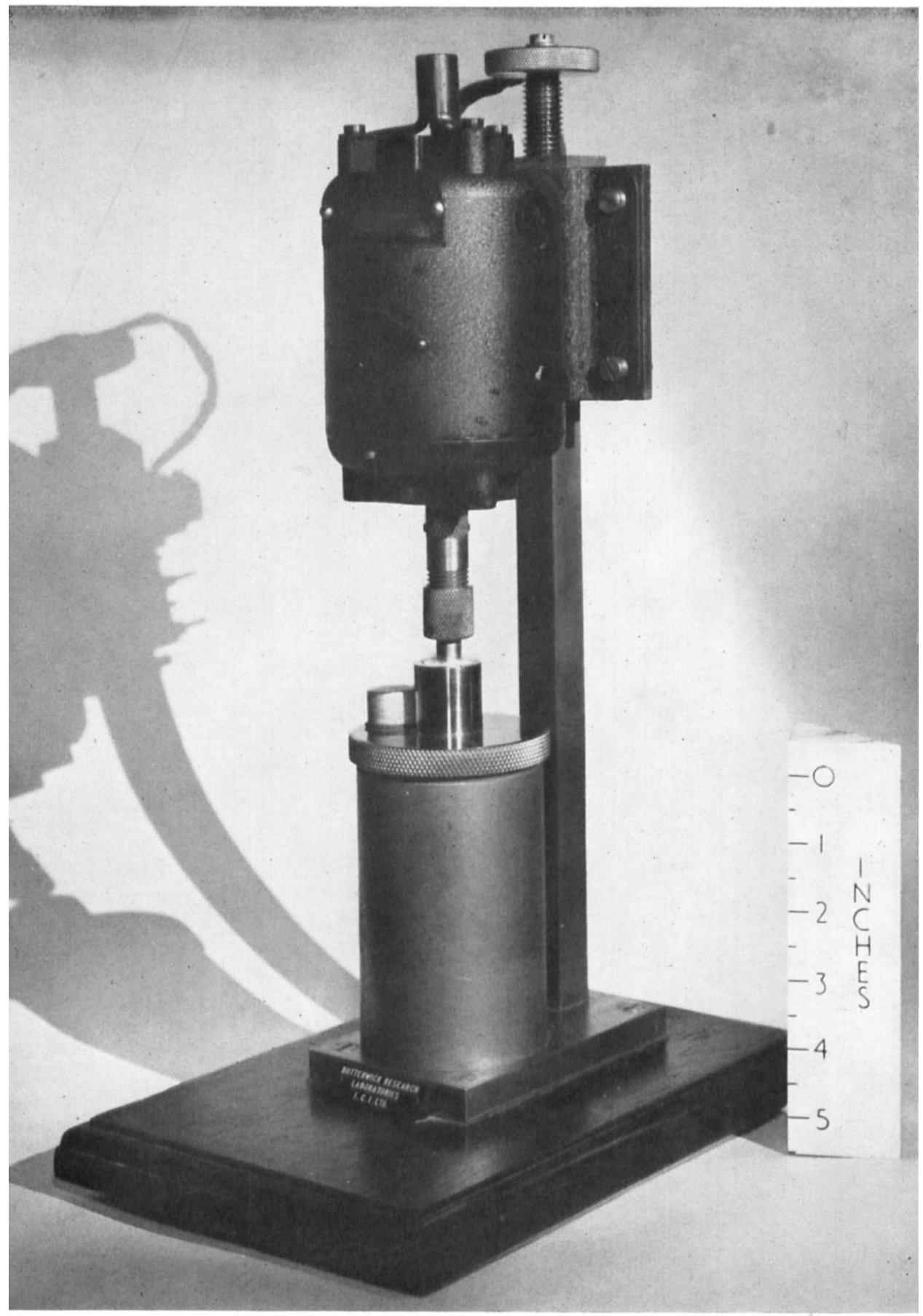

W. T. Moore \& B. Mason-Blender for mycelial inocula. Plate 1 